\title{
Uso das tecnologias digitais para capacitação das equipes aéreas de saúde: gestão do conhecimento
}

RESUMO | Objetivo: Descrever a experiência do processo de criação e compartilhamento dos treinamentos em formato de vídeos para capacitar as equipes de transporte inter-hospitalar aeromédico, no início da pandemia. Método: Trata-se de um relato de experiência, sendo realizado em abril de 2021, sobre a criação de vídeos educativos com intuito de capacitar as equipes no atendimento de casos suspeitos ou confirmados de COVID-19. Resultados: Os vídeos foram elaborados pela equipe multiprofissional, no início da pandemia. A capacitação por 'Ensino a Distância Emergencial' tornou-se a melhor escolha. Foram elaborados 10 vídeos, com tempo médio de elaboração de 639 minutos, utilizada a plataforma digital do YouTube®, disponibilizados para equipes, que aderiram 100\%. Conclusão: Fica evidente a importância da qualificação, com a finalidade de reconhecer efetivamente as particularidades do atendimento no ambiente aeroespacial, alinhados a gestão do conhecimento Descritores: COVID-19; Gestão do Conhecimento; Transporte Aeromédico; Vídeos educativos; Relato de caso.

ABSTRACT | Objective: To describe the experience of the process of creating and sharing training in video format to train air medical inter-hospital transport teams at the beginning of the pandemic. Method: This is an experience report, carried out in April 2021, on the creation of educational videos to train teams in the care of suspected or confirmed cases of COVID-19. Results: The videos were prepared by the multidisciplinary team at the beginning of the pandemic. 'Emergency Distance Learning' training became the best choice. Ten videos were produced, with an average time of 639 minutes, using the YouTube ${ }^{\circledR}$ digital platform, made available to teams, which adhered $100 \%$. Conclusion: The importance of qualification is evident, in order to effectively recognize the particularities of service in the aerospace environment, in line with knowledge management.

Descriptors: COVID-19; Knowledge management; Aeromedical Transport; Educational videos; Case report.

RESUMEN | Objetivo: Describir la experiencia del proceso de creación y puesta en común de capacitaciones en formato de video para capacitar a equipos de transporte aéreo médico interhospitalario al inicio de la pandemia. Método: Se trata de un informe de experiencia, realizado en abril de 2021, sobre la creación de videos educativos con el fin de capacitar a los equipos en la atención de casos sospechosos o confirmados de COVID-19. Resultados: Los videos fueron elaborados por el equipo multidisciplinario al inicio de la pandemia. La formación "Aprendizaje a distancia de emergencia" se convirtió en la mejor opción. Se produjeron diez videos, con un tiempo promedio de 639 minutos, utilizando la plataforma digital YouTube®, puestos a disposición de los equipos, que se adhirieron al 100\%. Conclusión: La importancia de la calificación es evidente, para reconocer efectivamente las particularidades del servicio en el entorno aeroespacial, en línea con la gestión del conocimiento

Descriptores: COVID-19; Conocimiento administrativo; Transporte Aeromédico; Videos educativos; Reporte de un caso.

\section{Bruno Gonçalves da Silva}

Mestre em Administração de Empresas (Centro Universitário UNA), Doutorando em Sistemas de Informações e Gestão do Conhecimento (Universidade FUMEC). Professor Assistente FCMMG e Enfermeiro de Bordo da Unimed Aeromédica

ORCID: 0000-0001-5173-0036

\section{Vânia Paula de Carvalho}

Enfermeira (PUC-MINAS), Enfermeira Intensivista (IEC-PUC MG) e Enfermeira Aeroespacial (FIC-UNIVIRTUAL), Mestre em Promoção da Saúde e Prevenção da Violência (UFMG). Coordenadora de Enfermagem/RT da Unimed Aeromédica.

ORCID: 0000-0002-9336-3606

\section{Maria Eduarda Becho Arger Marchetti}

Médica (UFMG), Especialista em Anestesiologista e Medicina Aeroespacial. Médica
Emergencista e Horizontal na UPA-CS BH, Anestesista em HCMG e Médica de Bordo na Unimed Aeromédica

ORCID: 0000-0002-0242-243X

\section{André Alves Elias}

Médico (UNIFENAS). Médico de Bordo na Unimed Aeromédica e BOA-SAMU BH ORCID:0000-0002- 1103-6450

\section{Flávio Lopes Ferreira}

Médico (UFMG), Mestre em Fisiologia e Farmacologia (UFMG), Cirurgião Geral e Especialista em Medicina Aeroespacial. Gestor Médico da Unimed Aeromédica, Professor na Faculdade de Ciências Médicas de Minas Gerais

ORCID: 0000-0001-7740-4394

\section{Armando Sérgio de Aguiar Filho}

Comunicador Social, Professor Titular na Uni- versidade FUMEC e da Faculdade Promove. Doutor em Gestão da Informação e do Conhecimento (UFMG) ORCID: 0000-0001-5542-7165
Recebido em: 29/09/2021

Approved: 08/10/2021 
INTRODUÇÃO

$\mathrm{N}$ o final de 2019, no continente asiático, foi identificado um vírus denominado Coronavírus (COVID -19), que causa infecções respiratórias e possui uma alta taxa de transmissibilidade, modificando o cotidiano no mundo.1 Para os profissionais de saúde, em particular, os procedimentos geradores de aerossóis foram os grandes fomentadores do contágio e faziam parte integrante da rotina de assistência. 2

Nesta perspectiva, por questão de segurança para as equipes assistenciais, foram aplicados os protocolos de distanciamento de acordo com a realidade do serviço e a capacitação por 'Ensino a Distância Emergencial' (EaD)3 tornou-se uma ferramenta indispensável para os treinamentos, não faziam parte do escopo e foram adaptados para essa nova realidade.

A cultura, o ambiente organizacional influencia e envolve as pessoas, os sistemas, os processos e, consequentemente, moldam a gestão do conhecimento. Segundo pesquisas, o compartilhamento do conhecimento pode ser produtivo e ser fundamentado em respeito mútuo e confiança. 4,5

A presente experiência começou com a discussão entre um grupo de enfermeiros e médicos especialistas em transporte aéreo de pacientes criticamente enfermos. E a questão norteadora surgiu: Como capacitar a equipe para atendimento aos pacientes de casos suspeitos ou confirmados de COVID- 19, sem infringir as regras de isolamento?

Sendo assim, esse relato de experiência tem por objetivo de descrever a experiência do processo de criação e compartilhamento dos treinamentos em formato de vídeos para capacitar as equipes de transporte inter-hospitalar aeromédico, no início da pandemia.
A cultura,

o ambiente

organizacional influencia e envolve as pessoas, os sistemas, os processos e, consequentemente, moldam a gestão do conhecimento. Segundo pesquisas, o compartilhamento do conhecimento pode ser produtivo e ser fundamentado em respeito mútuo e confiança
Tipo de estudo

Trata-se de um relato de experiência, com abordagem descritiva, sobre a criação de vídeos educativos com intuito de capacitar as equipes no atendimento de casos suspeitos ou confirmados de COVID-19, por meio de vídeos compartilhados em plataformas digitais.

A pesquisa descritiva manifesta as características da população específica ou do fenômeno que será estudado, conceitua a sua natureza e faz analogia entre as variáveis. "Não tem o compromisso de explicar os fenômenos que descreve, embora sirva de embasamento para tal explicação".6 Os registros de imagem foram realizados durante as gravações, pelos autores.

\section{Cenário}

Este relato de experiência é proveniente de uma empresa privada que presta serviços especializados de transporte inter-hospitalar aeromédico a usuários de saúde, sediada em Belo Horizonte, no Estado de Minas Gerais, Brasil.

\section{Critério de seleção}

Foi selecionada a experiência exitosa de um grupo de colaboradores da empresa de transporte aeromédico, que realizaram a capacitação das equipes por intermédio da plataforma digital do YouTube ${ }^{\circ}$ e respeitaram os protocolos de segurança, no início da pandemia.

\section{Coleta dos dados}

Foram necessárias uma série de cuidados para proteção de todos os profissionais de saúde e caberia a cada membro da equipe o entendimento dos perigos envolvidos e, mais do que nunca, a correta execução e o cuidado mútuo com todos os envolvidos na condução dos procedimentos, no início da pandemia.

As tarefas foram divididas de acordo com a habilidade pessoal, sendo: 
direção, atuação, produção, filmagem, roteiro, edição e revisão. Sendo que, os vídeos foram realizados no mês de março e disponibilizados para equipe no início do mês de abril, do ano de 2020.

Desta maneira, um grupo de cinco colaboradores especialistas, criaram vídeos educativos, demonstraram os procedimentos recomendados pelas diretrizes internacionais e nacionais, para proteção individual e melhor atendimento de pacientes com suspeita ou casos confirmados de infecção por coronavírus.

\section{Análise dos dados}

Os vídeos foram editados por um profissional enfermeiro do grupo de especialistas que possui expertise em processos de editoração de mídias. O trabalho iniciou-se com os arquivos brutos, sendo ajustado roteiro, coloração, áudio e inserção de legendas para melhor entendimento das equipes. Após edição, foi revisado por uma profissional enfermeira da equipe garantindo a qualidade dos processos e procedimentos. A divulgação das imagens foi autorizada pelos participantes por meio da assinatura do termo de autorização do uso de imagens.

\section{Aspectos Ético}

Trata-se do relato de experiência, realizado por intermédio de simulações realísticas, não houve pesquisa envolvendo seres humanos e as

informações de domínio público. Sendo assim, não houve necessidade de apreciação por Comitê de Ética em Pesquisa.

\section{RESULTADOS}

Dos cinco colaboradores responsáveis pela elaboração dos vídeos educativos participaram três enfermeiros e dois médicos. Houve a colaboração de dois assistentes de pista para que pudessem compor todos os atores do
Fluxograma 1. Descrição para a produção dos vídeos.
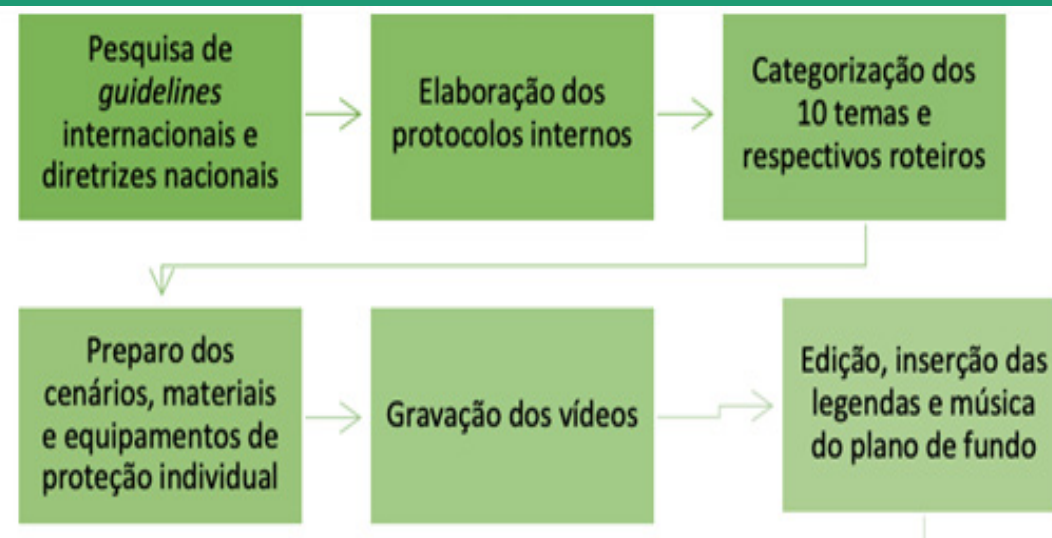

\section{Correção, validação}

e divulgação

Fonte: Elaborado pelos autores, 2021.

\section{Table 1. Titles of studies, categories covered and years that were published.}

Vídeos disponibilizados para capacitação por EaD - COVID-19

Tempo/minutos

Higienização das mãos com álcool 70\%

270

Paramentação da equipe médica e dos pilotos, com uso do macacão com capuz para proteção de operações com risco biológico, botas e EPI's*

Desparamentação da equipe médica e dos pilotos, com uso do macacão com capuz para proteção de operações com risco 2160 biológico, botas e EPI's*

4 Paramentação com capote impermeável e EPI's* para os assistentes de pista

\begin{tabular}{|ccc|}
\hline 5 & $\begin{array}{c}\text { Desparamentação do capote impermeável } \\
\text { e EPI's* para os assistentes de pista }\end{array}$ & 360 \\
\hline 6 & Desinfeção Maca na fase pós voo & 360 \\
\hline 7 & Desinfecção aeronave na fase pós voo & 540 \\
\hline 8 & Técnica da punção de cricotireoidostomia & 540 \\
\hline 9 & $\begin{array}{r}\text { Utilização de drogas para intubação orotra- } \\
\text { queal (sequência rápida) }\end{array}$ & 540 \\
\hline & $\begin{array}{r}\text { Cuidados para o preparo e o procedimento } \\
\text { de intubação orotraqueal }\end{array}$ & 540 \\
\hline
\end{tabular}


Tempo médio/minutos

Total para elaboração/minutos

*EPI's: Equipamentos de proteção individual

Fonte: Elaborado pelos autores, 2021.

Figura 1- Paramentação da equipe médica e dos pilotos, com uso do macacão com capuz para proteção de operações com risco biológico, botas e equipamentos de proteção individual.

Figura 2- Cuidados para preparo da intubação orotraqueal, com uso de capote impermeável e equipamentos de proteção individual.

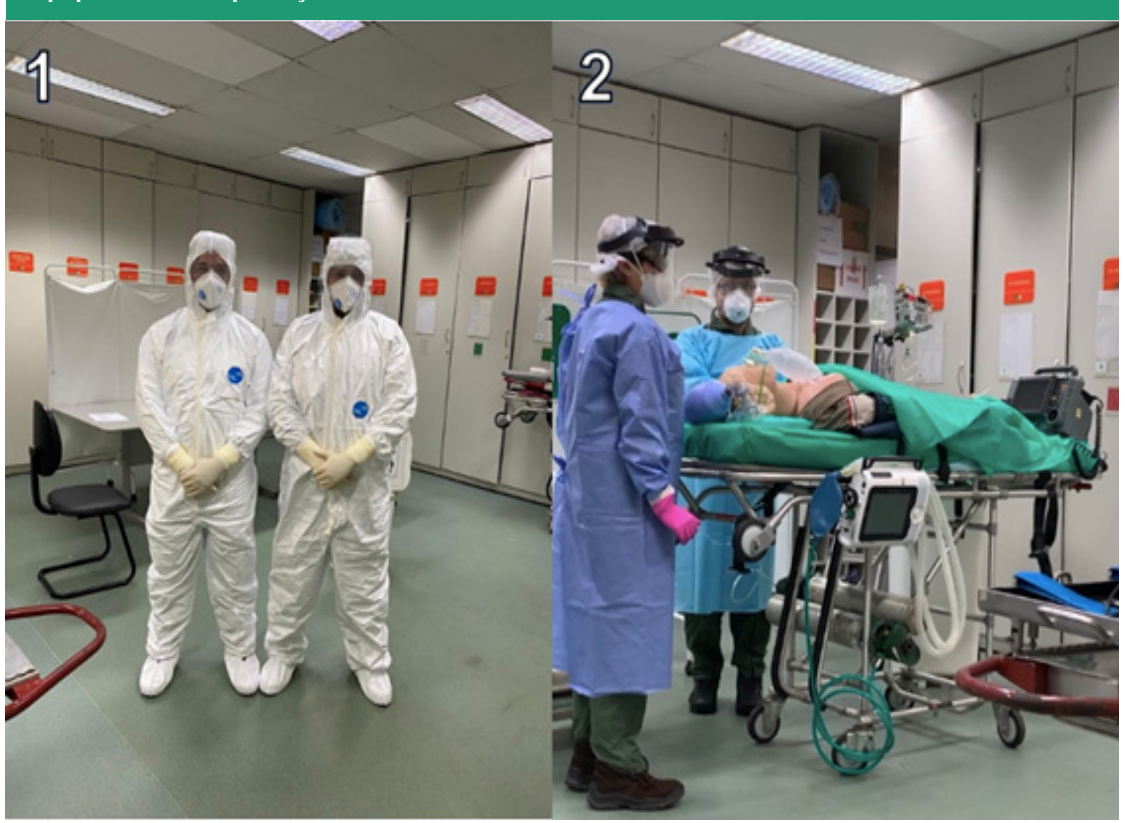

Fonte: Elaborado pelos autores, 2021.

processo de atendimento.

A equipe multiprofissional se uniu e deu início ao projeto de criar conteúdos digitais, pois havia uma restrição de treinamentos presenciais no período inicial da pandemia. Dessa forma, a capacitação por 'Ensino a Distância Emergencial' (EaD) tornou-se uma ferramenta valiosa. 3

Os vídeos técnicos desenvolvidos, possuem o foco no atendimento dos pacientes suspeitos ou confirmados com COVID-19 e para sua produção houve os passos informados no fluxograma 1.

Diante do exposto, os colaboradores participaram efetivamente de quase todos os processos, exceto da edição. Os vídeos foram, exclusivamente, editados por um profissional enfermeiro,

\section{.}


mizar o domínio dos conhecimentos de práticas avançadas para as equipes multiprofissionais.9-10 Sendo extremamente importante que sejam realizadas novas pesquisas que a inovem e preencham a lacuna de saberes no contexto da saúde, considerando as diferentes perspectivas.

\section{CONCLUSÃO}

Fica evidente a importância da qualificação, com a finalidade de reconhecer efetivamente as particularidades do atendimento no ambiente aeroespacial e logo, aprimorar a qualidade ao atendimento no transporte aeromédico, alinhados a gestão do conhecimento.

Podemos concluir assim, que líderes transformadores incentivam os colaboradores a darem importância para assimilar os novos processos e a se interessarem por melhorar suas qualificações, otimizarem suas práticas e alcançar os objetivos organizacionais.

Diante disso, esse relato de experiência teve o objetivo de descrever a experiência do processo de criação e compartilhamento dos treinamentos em formato de vídeos para capacitar as equipes de transporte inter-hospitalar aeromédico, no início da pandemia.

Foram elaborados 10 vídeos, com tempo médio de elaboração de 639 minutos, utilizada a plataforma digital do YouTube ${ }^{\circledR}$, disponibilizados para equipes, que aderiram $100 \%$.

Reiteramos que, há necessidade da realização de novos estudos para que possamos ampliar nossos conhecimentos à cerca da gestão do conhecimento voltadas para o campo da saúde, em especial, no campo da medicina aeroespacial.

\section{Referências}

1. Bascetta, T., Bolton, L., Kurtzman, E., Hantzos, W., Standish, H., Margarido, P., ... \& Gluck, J. (2021). Air Medical Transport of Patients Diagnosed With Confirmed Coronavirus Disease 2019 Infection Undergoing Extracorporeal Membrane Oxygenation: A Case Review and Lessons Learned. Air medical journal, 40(2), 130-134.

2. Matte, D. L., Cacau, L., da Fonseca Reis, L. F., \& Assis, M. C. (2020). Recomendações sobre o uso de equipamentos de proteção individual (EPIs) no ambiente hospitalar e prevenção de transmissão cruzada na COVID-19. ASSOBRAFIR Ciência, 11(Suplemento 1), 47-64.

3. dos Santos Rosenau, L., Dÿck, M. S., Pegorini, D. G., \& Urbanetz, S. T. (2020). Seria a educação a distância a resposta para uma época de pandemia?. Revista Observatório, 6(3), a15pt-a15pt.

4. Sordi, V. F., Nakayama, M. K., \& Binotto, E. (2018). Compartilhamento de conhecimento nas organizações: Um modelo analítico sob a ótica da ação cooperativa. Perspectivas em Gestão \& Conhecimento, 8(1), 44-66.

5. Carneiro, H. L. B. (2019). Percepções do impacto da cultura organizacional na gestão do conhecimento para a inovação de processos em serviços.

6. Vergara, S. C. (2006). Projetos e relatórios de pesquisa. São Paulo: Atlas

7. Bonin, W. L. M. (2016). Estratégia de educação para o apoio aeromédico: um estudo descritivo.

8. Salles, V. O., \& Matos, E. A. S. A. (2017). A teoria da complexidade de Edgar Morin e o Ensino de Ciência e Tecnologia. Revista Brasileira de Ensino de Ciência e Tecnologia, 10(01), 1-12.

9. Sibbald, S. L., \& Kothari, A. (2015). Creating, synthesizing, and sharing: The management of knowledge in public health. Public Health Nursing, 32(4), 339-348.

10. da Silva, B. G., Viana, L. L., Faustino, S. D. S. F., Silveira, C. D. P. S., de Carvalho, V. P., \& de Aguiar Filho, A. S. (2021). Preparação do enfermeiro para 0 atendimento à múltiplas vítimas no resgate aéreo. Nursing (São Paulo), 24(278), 5948-5957. 